Contents lists available at www.ijpba.in

International Journal of Pharmaceutical and Biological Science Archive

PubMed (National Library of Medicine ID: 101738825)

Index Copernicus Value 2017: 71.80

Volume 7 Issue 4; July-August; 2019; Page No. 01-10

\title{
A REVIEW ON ANALYTICAL METHOD DEVELOPMENT AND VALIDATION OF CALCIUM CHANNEL BLOCKERS AND ANGIOTENSIN- CONVERTING ENZYME INHIBITORS IN BULK AND PHARMACETICAL FORMULATION
}

\author{
Gawade Sonba. C, G.K. Dyade, Dr.S.G.Jadhav.
}

S.V.P.M.s College of Pharmacy, Malegaon (BKII) Baramati, Pune-600117

Conflicts of Interest: Nil

Corresponding author: Gawade Sonba. C

\section{ABSTRACT}

A simple, economical and rapid by UV detector and PDA Detector was used for Estimation of Trandolapril and Verapamil in combination and other drugs in various Pharmaceutical formulation. Calcium channel blockers(CCBs) and angiotensin- converting enzyme (ACE) inhibitors has been developed and fully validated by High performance liquid Chromatographic Methods. Calcium channel blockers (CCBs) or Calcium antagonists are among the most widely used drugs in cardiovascular medicine and hypertension also in angina. CCBs promote vasodilator activity by reducing calcium influx into vascular smooth muscle cells by interfering with calcium channels in the cell membrane. Trandolapril is a potent nonsulfhydryl and dicarboxyl containing Angiotensin converting inhibitor (ACE). Trandolapril used to treatment of hypertension appears to result the inhibition of tissue ACE activity and to improve survival myocardial infarction thereby reduce angiotensin II formation.

It includes drugs like Trandolapril, Norverapamil, Nifedipine, Verapamil. This Review enlists different method Developed, Validated and determination of Calcium channel blockers and angiotensin- converting enzyme inhibitors Like, RP-HPLC, LC-MS/MS and HPLC UV- Spectophotometric method. This method was also validated for various validation terms indicates that precise, accurate, linearly, and limit of Detection and limit of Quantitation as per ICH guidelines.

Keywords: HPLC Chromatography, Calcium Channel blocker, angiotensin- converting enzyme (ACE) inhibitors, Hypertension, Validation etc.

\section{INTRODUCTION}

Trandolapril is a colorless and crystalline solid soluble in chloroform, methanol and dichloromethane, odourless powder which melts in the range of $125-130 \circ \mathrm{C}$. Trandolapril is chemically (2S, 3aR, 7aS)-1- [(S)-2- [[1-Ethoxycarbony I-3phenylpropyl] amino] propanoyl] octahydro- $1 \mathrm{H}-$ indole- 2- carboxylic acid ${ }^{[6,7,8]}$. Molecular formula and molecular weight of the trandolapril drug are $\mathrm{C}_{24} \mathrm{H}_{34} \mathrm{~N}_{2} \mathrm{O}_{5}$ and 430.537 grams $/ \mathrm{mol}$ respectively ${ }^{[4,5]}$. Monoester prodrug of a Trandolapril was hydrolysed by esterases to its active dicarboxylic acid metabolite in syntehsis converted to the Trandolaprilat ${ }^{[9]}$. Verapamil is solid freely soluble in water, chloroform and methanol which melts range of $138-140{ }^{\circ} \mathrm{C}$. Verapamil hydrochloride (VER) is Chemically, (5- $[3,4-$ dimethoxyphenethyl) methylamino] -2- (3,4- dimethoxyphenyl)- 2isopropylvaleronitrile hydrochloride), a slow calcium channel antagonist, inhibits the trans membrane influx of calcium ions into the heart and vascular smooth muscle cells ${ }^{(6)}$. Verapamil is available in oral and intravenous dosage forms. Verapamil appears to be well absorbed orally, is highly protein bound, and is extensively metabolized by the liver to an active demethylated metabolite, norverapamil ${ }^{(7)}$.

Amphoteric compounds like trandolapril is a potent nonsulfhydryl and dicarboxyl containing Angiotensin converting inhibitor(ACE). Trandolapril used to treatment of hypertension appears to result the inhibition of tissue ACE activity and to improve survival myocardial infarction thereby reduce angiotensin II formation, and treatment for congestive heartfailure, decreases the rate of aldosterone secretion, and incease plasma renin. Decreased aldosterone secretion leads to diuresis, natriuresis, and a small rate of change of serum potassium. Some undesirable effects shows commonly used to treatment of trandolapril includes, dizziness, cough, headache. Approximately $10 \%$ and $70 \%$ oral dose of trandolapril is bioavailable as trandolapril and trandolaprilat 
respectively. The $t 1 / 2$ of trandolapril is maximum 1 hours, and that of Trandolaprilat is, approximately, 75 hours $^{[5,6]}$. Calcium channel blockers (CCBs) are a structurally and functionally heterogeneous group of medications that are used widely to control blood pressure and manage symptoms of angina. CCBs are particularly effective against large vessel stiffness, one of the common causes of elevated systolic blood pressure in elderly patients ${ }^{(8)}$. calcium channel blocking agents useful in the treatment of vasospastic angina, chronic stable angina, and supraventricular tachyarrhythmias ${ }^{(7)}$.

Advantages of CCBs are:

- Do not compromise haemodynamic: No impairment of physical work capacity

- No sedation or other CNS effect, cerebral perfusion is maintained: compatible with intense mental activity

Table 1: Analysis of Trandolapril and Verapamil combination with other drugs by RP-HPLC Method and UV-Spectrophotometric Mthods

\begin{tabular}{|c|c|c|c|c|}
\hline Sr. No. & Drug & Method & Description & ef \\
\hline 1. & $\begin{array}{l}\text { Development and } \\
\text { Validation of } \\
\text { Trandolapril in } \\
\text { Tablets }\end{array}$ & $\begin{array}{l}\text { RP-HPLC Method } \\
\text { with UV- Detector }\end{array}$ & 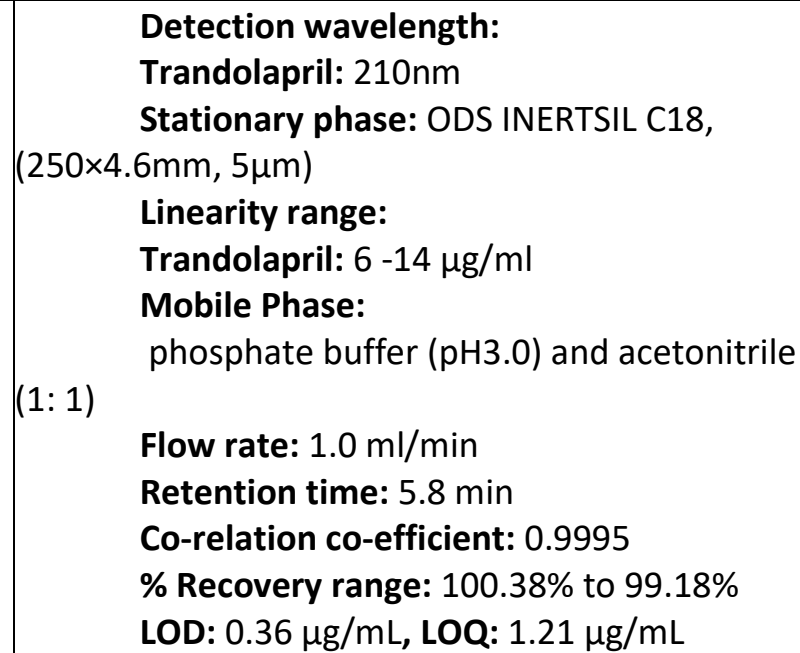 & \\
\hline & $\begin{array}{l}\text { Quantification of } \\
\text { Trandolapril }\end{array}$ & $\begin{array}{l}\text { UV- Spectrometric } \\
\text { Detection }\end{array}$ & $\begin{array}{l}\text { Detection wavelength: } \\
\text { Trandolapril: } 220 \mathrm{~nm} \\
\text { Stationary phase: LiChroCART -RP C18 } \\
\text { column (250x4.0, } 5 \mu \mathrm{m}) \\
\text { Linearity range: } \\
\text { Trandolapril: } 2.5-17.5 \mu \mathrm{g} / \mathrm{mL} \\
\text { Mobile Phase: acetonitrile: methanol: } \\
\text { phosphate buffer (0.025mM) pH3.0 (40:35:25) } \\
\text { Flow rate: } 1.0 \mathrm{ml} / \mathrm{min} \\
\text { Retention time: } 2.750 \pm 0.008 \mathrm{~min} \\
\text { Co-relation co-efficient: } 0.999 \\
\text { \% Recovery range: } 100.38 \% \text { to } 99.18 \% \\
\text { LOD: } 0.099 \mu \mathrm{g} / \mathrm{mL}, \text { LOQ: } 0.300834 \mu \mathrm{g} / \mathrm{ml}\end{array}$ & 0 \\
\hline
\end{tabular}

- Do not affect male sexual function, deleterious effect on plasma lipid profile, uric acid level and electrolyte balance.

Advantages of ACE Inhibitors are:

- The therapy of hypertension and heart failure.

- Trandolapril is associated with a low rate of transient serum amino transferase elevations, but has yet to be linked to instances of acute liver injury.

- This prevents the potent vasocontrictive action of angiotensin II and result in vasodilation

Reported methods are categorized depending on the following considerations:

Analyzed by Single component with other class drugs for combination with Calcium channel blocker with angiotensin- converting enzyme (ACE) inhibitors by UV-Spectroscopy methods and Chromatographic method. 


\begin{tabular}{|c|c|c|c|c|c|}
\hline & $\begin{array}{l}\text { Estimation of } \\
\text { Verapamil and } \\
\text { Trandolapril in } \\
\text { Pharmacetical } \\
\text { formulations tablets }\end{array}$ & $\begin{array}{l}\text { Liquid } \\
\text { Chromatographic } \\
\text { (RP-LC) Method }\end{array}$ & $(250 \times 4$ & $\begin{array}{l}\text { Detection wavelength: } \\
\text { Verapamil: } 202 \mathrm{~nm} \\
\text { Trandolapril: } 206 \mathrm{~nm} \\
\text { Stationary phase: X-Terra RP-18 column } \\
\text { t.60 mm } \times 5 \mu \mathrm{m} \text { ) } \\
\text { Linearity range: } \\
\text { Verapamil: } 0.50-18.00 \mu \mathrm{g} / \mathrm{mL} \\
\text { Trandolapril: } 0.05-1.00 \mu \mathrm{g} / \mathrm{mL} \\
\text { Mobile Phase: } \mathrm{MeOH} \text { and water (50-65) } \\
\text { Flow rate: } 1.2 \mathrm{ml} / \mathrm{min} \\
\text { Injected volume: } 20 \mu \mathrm{L} \\
\text { Retention time: } \\
\text { Verapamil: } 2.964 \mathrm{~min} \\
\text { Trandolapril: } 5.497 \mathrm{~min} \\
\text { Co-relation co-efficient: } \\
\text { Verapamil: } 0.9999 \\
\text { Trandolapril: } 0.9999 \\
\% \text { Recovery range: } \\
\text { Verapamil: } 99.99 \%, \text { Trandolapril: } 101.60 \\
\text { Verapamil : } \\
\text { LOD: } 0.008 \mu \mathrm{g} / \mathrm{mL}, \mathrm{LOQ}: 0.025 \mu \mathrm{g} / \mathrm{ml} \\
\text { Trandolapril: } \\
\text { LOD: } 0.018 \mu \mathrm{g} / \mathrm{mL}, \mathrm{LOQ}: 0.050 \mu \mathrm{g} / \mathrm{ml}\end{array}$ & \\
\hline 4. & $\begin{array}{l}\text { Determination of } \\
\text { Trandolapril in bulk } \\
\text { and formulations }\end{array}$ & RP-HPLC Method & $\begin{array}{l}215 \mathrm{~nm} \\
\mathrm{C} 18(100 \\
(50: 50) \\
\end{array}$ & $\begin{array}{l}\text { Detection wavelength: Trandolapril: } \\
\text { Stationary phase: Hypersil gold } \\
\text { Omm, } 4.6 \mathrm{~mm} \text {-ID, } 5 \mu \mathrm{m}) \\
\text { Linearity range: } \\
\text { Trandolapril: } 25.0-150 \mu \mathrm{g} / \mathrm{mL} \\
\text { Mobile Phase: buffer and acetonitrile } \\
\text { v/v } \\
\text { Flow rate: } 1.0 \mathrm{ml} / \mathrm{min} \\
\text { Retention time: } 6 \mathrm{~min} \\
\text { Co-relation co-efficient: } 0.9999 \\
\% \text { Recovery range: } 99.78 \% \text { to } 100.23 \\
\text { LOD: } 1.149 \mu \mathrm{g} / \mathrm{ml} ., \text { LOQ: } 3.832 \mu \mathrm{g} / \mathrm{ml} \text {. }\end{array}$ & 1 \\
\hline 5. & $\begin{array}{l}\text { Validation and } \\
\text { Determination of } \\
\text { Trandolapril in bulk } \\
\text { and formulations }\end{array}$ & $\begin{array}{l}\text { RP-HPLC Method } \\
\text { (PDA) }\end{array}$ & column & $\begin{array}{l}\text { Detection wavelength: } \\
\text { Trandolapril: } 210 \mathrm{~nm} \\
\text { Stationary phase: Hypersil-Gold C18 } \\
(250 \mathrm{~mm} \times 4.6 \mathrm{~mm}, 5 \mu \mathrm{m}) \\
\text { Linearity range: } \\
\text { Trandolapril: } 1-24 \mu \mathrm{g} / \mathrm{mL} \\
\text { Mobile Phase: acetonitrile and Buffer } \\
\text { lamine, pH } 3.0 \pm 0.1)(50: 50) \mathrm{v} / \mathrm{v} \\
\text { Flow rate: } 1.0 \mathrm{ml} / \mathrm{min} \\
\text { Retention time: } 4.6 \mathrm{~min} \\
\text { Co-relation co-efficient: } 0.9999 \\
\text { \% Recovery range: } 99 \% \\
\text { LOD: } 0.0566 \mu \mathrm{g} / \mathrm{ml} ., \text { LOQ } 0.1715 \mu \mathrm{g} / \mathrm{ml} \text {. }\end{array}$ & 2 \\
\hline
\end{tabular}




\begin{tabular}{|c|c|c|c|}
\hline $\begin{array}{l}\text { 6. } \\
\text { Simultaneous } \\
\text { estimation of Calcium } \\
\text { channel blockers in } \\
\text { API and dosage } \\
\text { formulations and } \\
\text { Human serum }\end{array}$ & $\begin{array}{l}\text { RP-HPLC Method } \\
\text { (UV-Detector) }\end{array}$ & $\begin{array}{l}\text { Detection wavelength: } \\
\text { Trandolapril: } 238 \mathrm{~nm} \\
\text { Stationary phase: Nucleosil }{ }^{\circledR} \mathrm{C} 18(10 \mu \mathrm{m} \text {, } \\
25 \times 0.46 \mathrm{~cm}) \text { column } \\
\text { Linearity range: } \\
\text { Verapamil: } 10-600 \mu \mathrm{g} / \mathrm{mL} \\
\text { Other Drugs: } 5-100 \mu \mathrm{g} / \mathrm{mL} \\
\text { Mobile Phase: } \mathrm{methanol}: \text { water: } \\
\text { acetonitrile }(55: 35: 10 \mathrm{v} / \mathrm{v} / \mathrm{v} ; \mathrm{pH} 2.65 \text { with OPA } \\
\text { Flow rate: } 1.0 \mathrm{ml} / \mathrm{min} \\
\text { Injected volume: } 20 \mu \mathrm{L} \\
\text { Run time: } 10 \mathrm{~min} \\
\text { Co-relation co-efficient: } 0.9998\end{array}$ & 3 \\
\hline 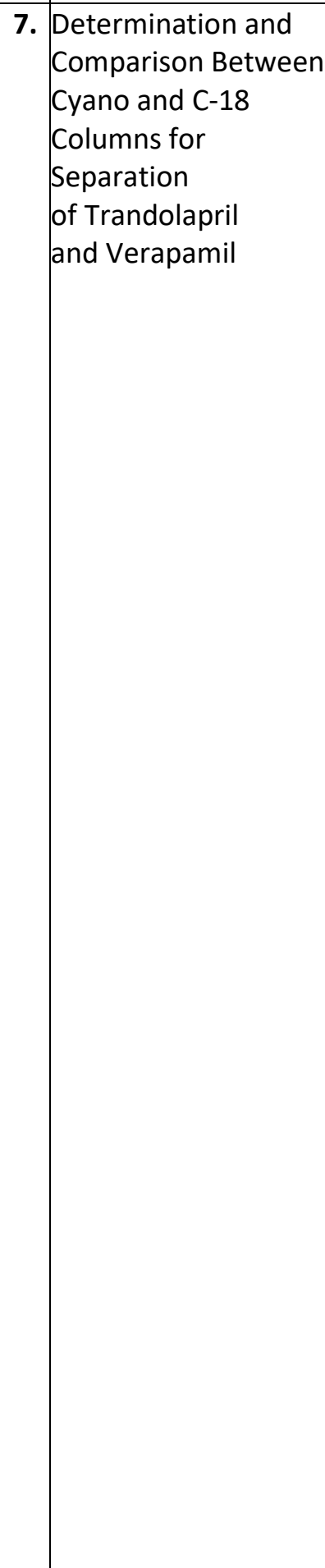 & $\begin{array}{l}\text { HPLC-UV Method } \\
\text { /LC-MS/MS }\end{array}$ & 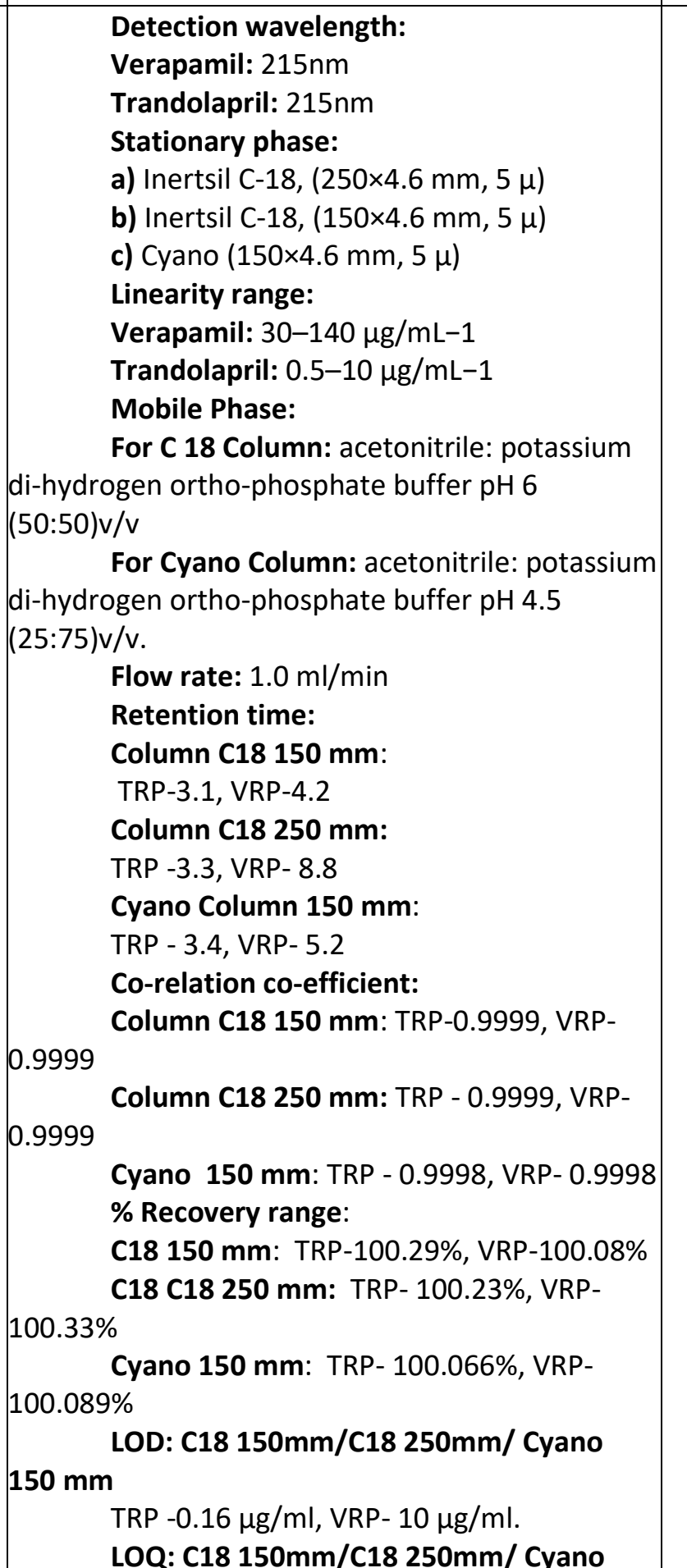 & 4 \\
\hline
\end{tabular}




\begin{tabular}{|c|c|c|c|c|c|}
\hline & & & $150 \mathrm{~mm}$ & TRP $-0.5 \mu \mathrm{g} / \mathrm{ml}$, VRP- $30 \mu \mathrm{g} / \mathrm{ml}$. & \\
\hline & $\begin{array}{l}\text { Verapamil and } \\
\text { Norverapamil in } \\
\text { Human Plasma }\end{array}$ & RP-HPLC Method & Wilmingt & $\begin{array}{l}\text { Detection wavelength: } \\
\text { Verapamil: } 201 \mathrm{~nm} \\
\text { Stationary phase: } \\
\text { Cyanopropylsilane column (Dupont, } \\
\text { ton, DE), } 15 \mathrm{~cm} \times 4.6 \mathrm{~mm} \\
\text { Linearity range: } \\
\text { Verapamil: } 20-100 \mu \mathrm{g} / \mathrm{mL} \\
\text { Mobile Phase: } \\
\text { acetonitrile and buffer (65:35\%)v/v } \\
\text { Flow rate: } 3.0 \mathrm{ml} / \mathrm{min} \\
\text { Injected volume: } 20 \mu \mathrm{L} \\
\text { Retention time: } \\
\text { Verapamil: } 3.92 \mathrm{~min} \\
\text { Co-relation co-efficient: } \\
\text { Verapamil: } 0.9935 \\
\text { \% Recovery range: } \\
\text { Verapamil: } 99.99 \% \\
\text { Verapamil : } \\
\text { LOD: } 2 \mathrm{ng} / \mathrm{ml}\end{array}$ & 5 \\
\hline & $\begin{array}{l}\text { Estimation of } \\
\text { Trandolapril Impurity } \\
\text { in API }\end{array}$ & $\begin{array}{l}\text { RP-UPLC-MS } \\
\text { Method }\end{array}$ & 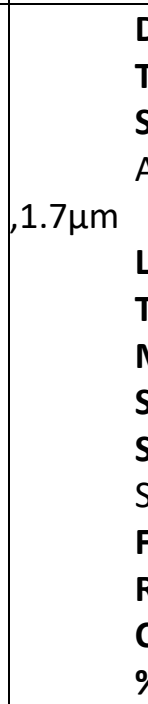 & $\begin{array}{l}\text { Detection wavelength: } \\
\text { Trandolapril: } 210 \mathrm{~nm} \\
\text { Stationary phase: } \\
\text { Acquity BEH C18, (100mm x } 2.1 \mathrm{~mm}) \\
\text { Linearity range: } \\
\text { Trandolapril: } 0.05 \text { to } 1.0 \% \\
\text { Mobile Phase: } \\
\text { Solution A : } 0.1 \% \text { TFA in water, } \\
\text { Solution B : } 0.1 \% \text { TFA in Acetonitrile. } \\
\text { Solution A : Solution B ( } 20: 80 \%) \\
\text { Flow rate: } 0.4 \mathrm{ml} / \mathrm{min} \\
\text { Retention time: } 5.56 \mathrm{~min} \\
\text { Co-relation co-efficient: } 0.9997 \\
\% \text { Recovery range: } 100 \text { to } 101.0 \%\end{array}$ & 6 \\
\hline & $\begin{array}{l}\text { Estimation of } \\
\text { Combination of } \\
\text { Trandolapril and } \\
\text { Verapamil in Bulk and } \\
\text { Pharmaceutical } \\
\text { formulation }\end{array}$ & $\begin{array}{l}\text { RP-HPLC Method } \\
\text { (UV-Detector) }\end{array}$ & 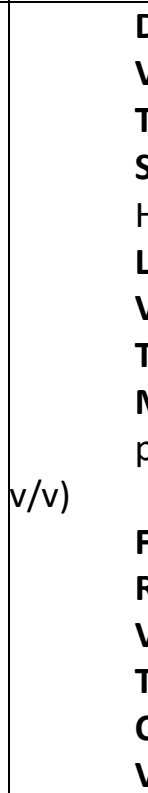 & $\begin{array}{l}\text { Detection wavelength: } \\
\text { Verapamil: } 240 \mathrm{~nm} \text {, } \\
\text { Trandolapril: } 240 \mathrm{~nm} \\
\text { Stationary phase: } \\
\text { Hypersil BDS C18 }(100 \mathrm{~mm} \times 4.6 \mathrm{~mm}, 5 \mu) \\
\text { Linearity range: } \\
\text { Verapamil: } 60-360 \mu \mathrm{g} / \mathrm{Ml} \text {, } \\
\text { Trandolapril: } 1-6 \mu \mathrm{g} / \mathrm{mL} \\
\text { Mobile Phase: } \\
\text { phosphate buffer and acetonitrile (60:40 } \\
\text { Flow rate: } 0.8 \mathrm{ml} / \mathrm{min} \\
\text { Retention time: } \\
\text { Verapamil: } 3.481 \mathrm{~min} \\
\text { Trandolapril: } 2.905 \mathrm{~min} \\
\text { Co-relation co-efficient: } \\
\text { Verapamil: } 0.9998 \text {, Trandolapril: } 0.9999\end{array}$ & 7 \\
\hline
\end{tabular}




\begin{tabular}{|c|c|c|c|c|}
\hline & & & $\begin{array}{l}\text { \% Recovery range: } \\
\text { Verapamil: } 99.65 \% \text {, Trandolapril: } 99.64 \% \\
\text { Verapamil : } \\
\text { LOD: } 4.923 \mu \mathrm{g} / \mathrm{mL} \text {, LOQ: } 14.918 \mu \mathrm{g} / \mathrm{ml} \\
\text { Trandolapril: } \\
\text { LOD: } 0.166 \mu \mathrm{g} / \mathrm{mL} \text {, LOQ: } 0.503 \mu \mathrm{g} / \mathrm{ml}\end{array}$ & \\
\hline $\begin{array}{l}\text { 11. Estimation of } \\
\text { Trandolapril in } \\
\text { Tablets dosage form }\end{array}$ & $\begin{array}{l}\text { RP-HPLC Method } \\
\text { (PDA detector) }\end{array}$ & $\begin{array}{l}\times 150 m \\
(35: 65\end{array}$ & $\begin{array}{l}\text { Detection wavelength: } \\
\text { Trandolapril: } 220 \mathrm{~nm} \\
\text { Stationary phase: Altima, } \mathrm{C} 18 \text { column }(4.6 \\
\mathrm{m}, 5 \mu \text { ) } \\
\text { Linearity range: } \\
\text { Trandolapril: } 6-36 \mu \mathrm{g} / \mathrm{mL} \\
\text { Mobile Phase: } \\
\text { phosphate buffer and acetonitrile } \\
\mathrm{v} / \mathrm{v} \\
\text { Column temperature: } 30^{\circ} \mathrm{C} \\
\text { Injection volume: } 10 \mathrm{ul} \\
\text { Flow rate: } 1.0 \mathrm{ml} / \mathrm{min} \\
\text { Retention time: } 2.9 \mathrm{~min} \\
\text { Run time: } 5 \mathrm{~min} \\
\text { Co-relation co-efficient: } 0.9999 \\
\text { \% Recovery range: } 100.13 \% \\
\text { LOD: } 0.28 \mu \mathrm{g} / \mathrm{ml} ., \mathrm{LQ}: 0.85 \mu \mathrm{g} / \mathrm{ml} .\end{array}$ & 8 \\
\hline $\begin{array}{l}\text { 12. Verapamil } \\
\text { Hydrochloride and } \\
\text { Trandolapril in bulk } \\
\text { and Their } \\
\text { Pharmaceutical } \\
\text { formulation }\end{array}$ & $\begin{array}{l}\text { RP-HPLC Method } \\
\text { (UV detection) }\end{array}$ & $\begin{array}{l}3.5 \mu) \\
(35: 65\end{array}$ & $\begin{array}{l}\text { Detection wavelength: } \\
\text { Verapamil: } 230 \mathrm{~nm} \text {, } \\
\text { Trandolapril: } 230 \mathrm{~nm} \\
\text { Stationary phase: } \\
\text { symmetrical C18 column }(4.6 \text { x } 150 \mathrm{~mm} \text {, } \\
\text { Linearity range: } \\
\text { Verapamil: } 10-65 \mu \mathrm{g} / \mathrm{Ml} \text {, } \\
\text { Trandolapril: } 2-15 \mu \mathrm{g} / \mathrm{mL} \\
\text { Mobile Phase: } \\
\text { Phosphate buffer (pH2.2):acetonitrile } \\
\text { v/v } \\
\text { Flow rate: } 0.6 \mathrm{ml} / \mathrm{min} \\
\text { Retention time: } \\
\text { Verapamil: } 2.5 \mathrm{~min} \\
\text { Trandolapril: } 3.8 \mathrm{~min} \\
\text { Co-relation co-efficient: } \\
\text { Verapamil: } 0.999, \text { Trandolapril: } 0.998 \\
\text { \% Recovery range: } \\
\text { Verapamil: } 98.44 \%, \text { Trandolapril: } 98.01 \% \\
\text { Verapamil : } \\
\text { LOD: } 0.018 \mu \mathrm{g} / \mathrm{mL}, \text { LOQ: } 0.06 \mu \mathrm{g} / \mathrm{ml} \\
\text { Trandolapril: } \\
\text { LOD: } 0.05 \mu \mathrm{gL} / \mathrm{mL} \text {, LOQ: } 0.19 \mu \mathrm{g} / \mathrm{ml}\end{array}$ & 9 \\
\hline $\begin{array}{l}\text { 13. Nifedipine and } \\
\text { Verapamil in Rat } \\
\text { Plasma }\end{array}$ & HPLC Method & 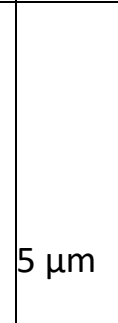 & $\begin{array}{l}\text { Detection wavelength: } \\
\text { Verapamil: } 235 \mathrm{~nm} \\
\text { Nifedipine: } 235 \mathrm{~nm} \\
\text { Stationary phase: } \\
\text { Microsorb-MV C18, }(25 \mathrm{~cm} \times 4.6 \mathrm{~mm}) \text { i.d., } \\
\text { Linearity range: }\end{array}$ & 0 \\
\hline
\end{tabular}




\begin{tabular}{|c|c|c|c|c|}
\hline & & $(\mathrm{pH} 5.2$ & $\begin{array}{l}\text { Verapamil: } 0.4-2 \mu \mathrm{g} / \mathrm{mL} \\
\text { Nifedipine: } 0.2-1 \mu \mathrm{g} / \mathrm{mL} \\
\text { Mobile Phase: } \\
\text { Acetonitrile: methanol: phosphate buffer } \\
0.01 \mathrm{M})(55: 15: 30) \\
\text { Flow rate: } 1.0 \mathrm{ml} / \mathrm{min} \\
\text { Retention time: } \\
\text { Verapamil: } 6.4 \mathrm{~min} \\
\text { Nifedipine: } 3.4 \mathrm{~min} \\
\text { Co-relation co-efficient: } \\
\text { Verapamil: } 0.998 \\
\text { Nifedipine: } 0.998 \\
\% \text { Recovery range: } \\
\text { Verapamil: } \\
\text { Nifedipine: }>97 \% \text {, (range }=97.9-98.3 \%) \\
\text { Verapamil : }>95 \% \text {, (range }=95.7-97.1 \% \text { ) }\end{array}$ & \\
\hline $\begin{array}{l}\text { 14.Trandolapril and } \\
\text { Verapamil in Human } \\
\text { Plasma }\end{array}$ & $\begin{array}{l}\text { Liquid } \\
\text { Chromatography } \\
\text { Tandem mass } \\
\text { Spectrometry }\end{array}$ & $\mathrm{mm}), 5 \mu$ & $\begin{array}{l}\text { Stationary phase: } \\
\text { waters symmetry-RP18 ( } 150 \mathrm{~mm} \times 4.0 \\
\text { Linearity range: } \\
\text { Verapamil: } 1-2000 \mu \mathrm{g} / \mathrm{Ml}, \\
\text { Trandolapril: } 5-1500 \mu \mathrm{g} / \mathrm{mL} \\
\text { Mobile Phase: } \\
\text { Ammonium formate }(10 \mathrm{mmol} \text { ) and } \\
\text { trile (70:30 \%) V/V } \\
\text { Flow rate: } 0.9 \mathrm{ml} / \mathrm{min} \\
\text { Co-relation co-efficient: } \\
\text { Verapamil: } 0.999, \text { Trandolapril: } 0.998 \\
\% \text { Recovery range: } \\
\text { Verapamil: } 98.37 \% \text {, Trandolapril: } 97.60 \%\end{array}$ & 1 \\
\hline $\begin{array}{l}\text { 15. Trandolaprl and } \\
\text { Verapamil } \\
\text { Hydrochloride in } \\
\text { Capsule Formulation }\end{array}$ & $\begin{array}{l}\text { Liquid } \\
\text { Chromatographic } \\
\text { Method }\end{array}$ & $\mu \mathrm{m})$ & $\begin{array}{l}\text { Detection wavelength: } \\
\text { Verapamil HCl: } 220 \mathrm{~nm} \text {, } \\
\text { Trandolapril: } 220 \mathrm{~nm} \\
\text { Stationary phase: } \\
\text { LiChrosorb RP-18 column }(250 \times 4 \mathrm{~mm}, 10 \\
\text { Linearity range: } \\
\text { Verapamil HCl: } 4-20 \mu \mathrm{g} / \mathrm{mL} \\
\text { Trandolapril: } 4-20 \mu \mathrm{g} / \mathrm{mL} \\
\text { Mobile Phase: } \\
\text { Acetonitrile: methanol: buffer (pH2.7) } \\
\text { O) v/v/v } \\
\text { Flow rate: } 1.0 \mathrm{ml} / \mathrm{min} \\
\text { Co-relation co-efficient: } \\
\text { Verapamil HCl: } 0.9996, \text { Trandolapril: } \\
\text { \% Recovery range: } \\
\text { Verapamil HCl: } 98.13 \% \text {, Trandolapril: }\end{array}$ & \\
\hline
\end{tabular}




\begin{tabular}{|c|c|c|c|}
\hline $\begin{array}{l}\text { 16. Determination of } \\
\text { Verapamil in } \\
\text { Pharmaceutical } \\
\text { formulation }\end{array}$ & HPLC Method & $\begin{array}{l}\text { Detection wavelength: } \\
\text { Verapamil: } 280 \mathrm{~nm} \\
\text { Stationary phase: } \\
\text { C18 column }(30 \mathrm{~cm} \times 4 \mathrm{~mm}), 10 \mu \mathrm{m} \\
\text { Linearity range: } \\
\text { Verapamil: } 0-274 \mu \mathrm{g} / \mathrm{mL} \\
\text { Mobile Phase: } \\
\text { Methanol: water: acetic acid: } \\
\text { triethylamine (55:44:1:0.1) } \\
\text { Flow rate: } 1.2 \mathrm{ml} / \mathrm{min} \\
\text { Injected volume: } 20 \mu \mathrm{L} \\
\text { Co-relation co-efficient: } \\
\text { Verapamil: } 0.9999 \\
\text { \% Recovery range: } \\
\text { Verapamil : } 100.0 \% \text { ( } 80 \mathrm{mg} \text { Tablets), } \\
101.0 \% \text { (120 mg Tablets) }\end{array}$ & 2 \\
\hline $\begin{array}{l}\text { 17. Determination of } \\
\text { Verapamil } \\
\text { Hydrochloride and its } \\
\text { Related compounds } \\
\text { in raw material }\end{array}$ & HPLC Method & 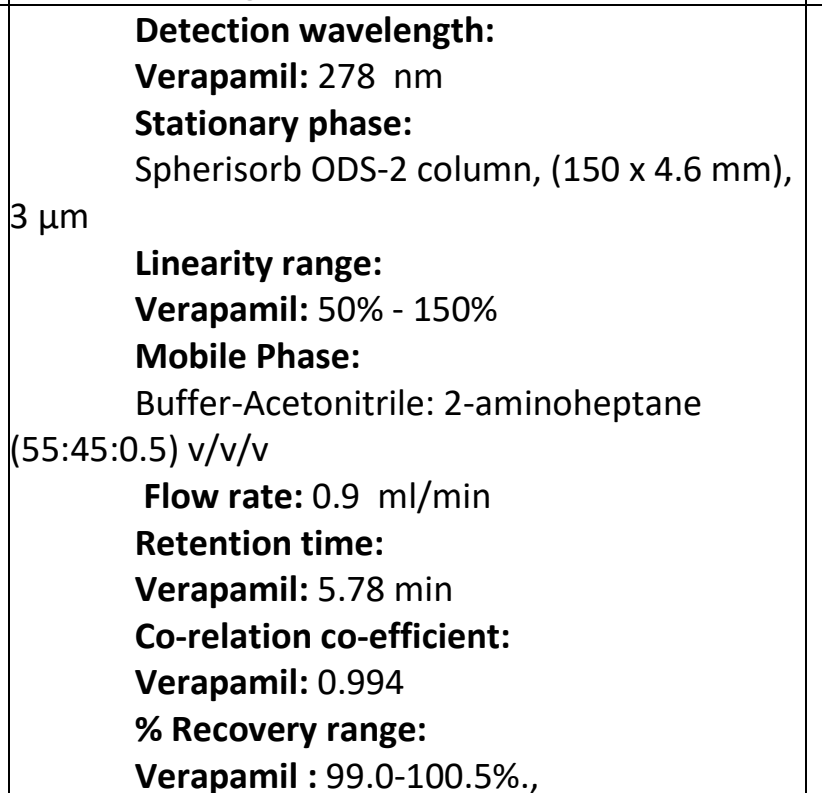 & 3 \\
\hline $\begin{array}{l}\text { 18. Comparative } \\
\text { pharmacokinetics of } \\
\text { trandolapril and its } \\
\text { active metabolite, } \\
\text { and verapamil in } \\
\text { human plasma }\end{array}$ & HPLC Method & $\begin{array}{l}\text { Detection wavelength: } \\
\text { Verapamil: } \mathrm{nm}, \\
\text { Trandolapril: } \mathrm{nm} \\
\text { Stationary phase: } \\
\text { Phenomenex } \mathrm{C} 18\left(3 \mu \mathrm{m}, 110 \mathrm{~A}^{\circ}, 100 \times 1\right. \\
\mathrm{mm}) \\
\text { Linearity range: } \\
\text { Verapamil: } 1.50-500 \mathrm{ng} \cdot \mathrm{mL}-1 \\
\text { Trandolapril: } 1.00-500 \mathrm{ng} \cdot \mathrm{mL}-1 \\
\text { Retention time: } \\
\text { Verapamil: } 5.51 \text { min } \\
\text { Nifedipine: } 6.61 \text { min } \\
\text { Mobile Phase: } \\
\text { phase A: } 2 \% \text { acetic acid (v/v) } \\
\text { phase B: } 90 \% \text { methanol and } 2 \% \text { acetic acid } \\
\text { (v/v) } \\
\text { Run Time: } 10 \text { min } \\
\text { Flow rate: } 50 \mu \mathrm{L} / \mathrm{min}, \text { gradient (30\% B } \\
\text { from } 0 \text { to } 1 \text { min, } 100 \% \text { B from } 1 \text { to } 3 \text { min, } 100 \% \text { B } \\
\text { from } 3 \text { to } 8 \text { min, } 30 \% \text { B from } 8 \text { to } 9 \text { min and } \\
\text { maintained at } 30 \% \text { till } 10 \text { min. }\end{array}$ & 4 \\
\hline
\end{tabular}




\begin{tabular}{|l|l|l|l|l|}
\hline & & & $\begin{array}{l}\text { Co-relation co-efficient: } \\
\text { Verapamil: } 0.9819, \text { Trandolapril: } 0.993\end{array}$ & \\
\hline
\end{tabular}

\section{CONCLUSION:}

This Review represents the Reported Spectrophotometric and Chromatographic Methods Developed and Validated for determination of Calcium channel blocker and angiotensinconverting enzyme (ACE) inhibitors in different Pharmaceuticals formulations. Here Calcium channel blocker and angiotensin- converting enzyme (ACE) inhibitors shows the simple, accurate, precise method development and validate of the different drug formulations. The RPHPLC, and LC-MS/MS, UV- Spectrophotometric method etc.

\section{REFERENCES:}

1. D.P. Ip, G.S. Brenner, in: K. Florey (Ed.), Analytical Profiles of Drug Substances, Academic Press Orlando FL (1987) 207-243.

2. D.N. Franz, A.R. Gennaro (Ed.), Remington: The Science and Practice of Pharmacy, vol. II, 19th ed., Mack Publishing Company, Pennsylvania (1995) 951.

3. G.T. Warner, C.M. Perry, Ramipril: a review of its use in the prevention of cardiovascular outcomes, Drugs 2002 62(9) 1381-1405.

4. http://www.medicines.org.uk/EMC/medicine/ 8279/SPC/gopten/ accessed on 30/05/10.

5. A. Gumieniczek and H. Hopkala, "Development and validation of a Liquid Chromatographic method for the Determination of Trandolapril and Verapamil in Capsules" Journal of Liquid Chromatography \& Related Technologies(2001), 24:3, 393-400.

6. Mehmet gumustas, Senem sanli, Nurullah sanli and Sibel A. Ozkan, "Development and Validation of a Liquid Chromatographic Method for Concurrent Assay of Weakly Basic Drug Verapamil and Amphoteric Drug Trandolapril in Pharmaceutical Formulations" Journal of Food and Drug Analysis, Vol. 20, 2012, Pages 588-596

7. Scott M. Johnson \& Shoukry K. Wahba Khalil, "An HPLC Method for the Determination of Verapamil and Norverapamil in Human Plasma" Journal of Liquid Chromatography, (1987) 10:6, 1187-1201

8. Najma Sultana, Saeed Arayne, and Shabana Naz Shah, "Development and Validation for the Simultaneous Quantification of Prazosin, Amlodipine, Diltiazem and Verapamil in API,
Dosage Formulation and Human Serum by RPHPLC: Application to in vitro interaction studies" Sultana et al, Med chem (2014), Pages 4-12

9. A.S.K.Sankar, M.Alekhya, Suraj Sythana, Dr.V.Ravichandiran, "Analytical method development and validation of Trandolapril jn Tablets by RP- HPLC" 2013 Volume 1 Issue 2 Pages 119- 127.

10. Sunil Kumar Dubey, Swapnil Deshpande, Sandeep Kumar, Prashant Raut, Akash Kumar Jain, Rajeev J Mudakavi,"A High performance liquid Chromatographic method for quantification of Trandolapril using UV Spectrometric detection" IJBR 1(3) (2010) Pages 134-140.

11. C. Rambabu, G. Ramu, A. Biksham Babu1 and S.Venkata Rao, "Optimized and validated Reverse Phase HPLC Method for the Determination of Trandolapril in bulk and formulations" Vol.3, No.4 (2010), 777-782 ISSN: 0974-1496.

12. Leena A. Al-Hawash, Ashok K. Shakya, and Maher L. Saleem, "Stability Indicating HPLC Determination of Trandolapril in Bulk Drug and Pharmaceutical Dosage Forms" Hindawi Publishing Corporation International Journal of Analytical Chemistry Volume (2015), pages 8

13. Najma Sultana, Saeed Arayn and Shabana Naz Shah, "Development and Validation for the Simultaneous Quantification of Prazosin, Amlodipine, Diltiazem and Verapamil in API, Dosage Formulation and Human Serum by RPHPLC: Application to in vitro Interaction Studies" Med chem ISSN an open access journal (2014) Volume 4(12): Page 770-777.

14. A. Hemda, Ragaa Magdy, Maha Farouk, "Comparison Between Cyano and C-18 Columns for Separation of Trandolapril and Verapamil with ESI-Q-ToF-MS Characterization of Acidic and Basic Degradation Products: Stability Indicating Assay Methods" Pharmaceutical Analytical Chemistry Department, Faculty of Pharmacy, Ahram Canadian University, 4th Industrial Region (2018)

15. Scott M. Johnson \& Shoukry K. Wahba Khalil (1987): An HPLC Method for the Determination of Verapamil and Norverapamil in Human Plasma, Journal of Liquid Chromatography, 10:6, Page1187-1201 
16. B. Anjaneyulu Reddy, P. Radhakrishnanand, Md. Irshad Alam, J. Satish and Arjun Siliveri, " Method Development, Validation And Stability Estimation Of Trandolapril Assay And Its Organic Impurities By RP-UPLC-MS" world journal of pharmaceutical science (2018), Vol 7, Issue 1, Page 559-575.

17. E Tejashwi, S. Kiran Kumar and B. Siddartha, "Simultaneous estimation and validation of Trandolapril and Verapamil in bulk and pharmaceutical dosage form by RP-HPLC Method" Published in World Journal of Pharmacy and Pharmaceutical Sciences (2014), Vol 3, Issue 12, Page 652-662

18. ML. Lal Prasanth, and Sridhar Siddiraju, "RPHPLC Method Development and validation for the estimation of Trandolapril in Tablet dosage form" IJRPC (2015), 5(2), Page 368-372.

19. P. Laxmi Madhuri, Vusuvandla Geetha ,"Development and validation of RP-HPLC method for the simultaneous estimation of Verapamil hydrochloride and Trandolapril in bulk and pharmaceutical dosage forms" Asian Journal of Pharmaceutical Analysis and Medicinal Chemistry. (2016) 4(1), 38 - 46.

20. S. M. Shahriyar \& C. A. Lau-Cam (2000), "A simple HJPLC method with spectrophotometric detection for simultaneous assay of Nifedipine and Verapamil in Rat Plasma" Journal of Liquid
Chromatography \& Related Technologies (2000), 23:8, 1253-1265.

21. Yatha Ravi, B.Rajkamal, "An improved LCMS/MS method development and validation for the determination of Trandolapril and Verapamil in Human plasma" International Journal of Pharmacy and Pharmaceutical Sciences Vol 11, Issue 3, 2019, ISSN- 0975 1491.

22. Dimitri C. Tsilifonis, Karen Wilk, Robert Reisch Jr. \& Robert E. Daly (1985) High Performance Liquid Chromatographic Assay of Verapamil Hydrochloride in Dosage Formsk, Journal of Liquid Chromatography, 8:3, 499-511

23. Pauline M, Lacroix J, Graham and Edward G. Lovering, "High-performance liquid chromatographic method for the assay of verapamil hydrochloride and related compounds in raw material" Journal of Pharmaceutical \& Biomedical Analysis(1991), Vol. 9, Nos 10-12, 817-822

24. Ragaa Magdy, Ahmed H. El-Khatib , Ahmed Hemdan, Omar Abd Elaziz, Maha Farouk, Michael W. Linscheid, "Comparative pharmacokinetics of trandolapril, its active metabolite, and verapamil in human plasma of Egyptian population using HPLC-MS/MS" : m.linscheid@chemie.hu-berlin.de. 2093 7575, Fax: 0049 (0)30 20936985. 